\title{
Ventilación mecánica compartida: ¿Una herramienta fútil o una estrategia aceptable para superar la crisis?
}

\author{
Shared mechanical ventilation: A futile tool or an acceptable \\ strategy to overcome the crisis?
}

Ramos Matías', Placenti Alejandro', Pérez Susana Gabriela

\begin{abstract}
Different events can trigger health crises, where demand exceeds the response capacity of the health system. In the current context of the COVID-19 pandemic, one edge of this demand may be the need for ventilatory support. Among the strategies to deal with the problem, a new possibility arises: using a single ventilator for more than one patient until adequate resources arrive. This work reviews the available evidence, exposes the physical foundations of its operation and draws attention to the warnings.
\end{abstract}

\section{RESUMEN}

Diferentes eventos pueden desencadenar crisis sanitarias, donde la demanda excede la capacidad de respuesta del sistema de salud. En el contexto actual de la pandemia de COVID-19, una arista de esta demanda puede ser la necesidad de soporte ventilatorio. Dentro de las posibles estrategias para afrontar el problema, surge la posibilidad de utilizar un único ventilador para dar soporte a más de un paciente hasta que lleguen los recursos adecuados. Este trabajo pretende revisar la evidencia disponible, exponer los fundamentos físicos de su funcionamiento y llamar la atención sobre las advertencias.

\section{Key words:}

Mechanical ventilation, crisis, sanitary collapse, COVID-19

\section{Palabras clave:}

Ventilación mecánica, crisis, colapso sanitario, COVID-19

División Anestesia, Analgesia y Reanimación. Hospital de Clínicas José de San Martín, Universidad de Buenos Aires.

Fecha de recepción: 26 de marzo de 2020

Fecha de aceptación: 31 de marzo de 2020

\section{ORCID}

https://orcid.org/0000-0001-8955-5587

Correspondencia:

Ramos, Matías

Email: matt.plate@gmail.com 


\section{Introducción}

A nte situaciones de sobrecarga sanitaria, dependiendo de la causa, puede aumentar la demanda de ventilación mecánica a tal punto que la capacidad de respuesta del sistema sanitario sea insuficiente. Dado el caso, pueden plantearse dos estrategias alternativas: disminuir esta demanda o bien aumentar la capacidad ventilatoria del sistema de salud. En el primer caso, se puede aplicar una terapia más restrictiva para el acceso a la ventilación mecánica o incluso derivar pacientes a otras instituciones (en situaciones de colapso sanitario esta alternativa es poco realista). En el segundo caso, aparecen opciones como la ventilación manual (bagging), la adquisición de nuevos ventiladores o una opción poco explorada: compartir la ventilación mecánica (un ventilador con más de un paciente). Esta opción se experimentó en Italia y España ante el colapso del sistema de salud durante la actual pandemia de COVID-19.

Es claro que este tipo de estrategia ventilatoria no se presenta como una opción superadora para el soporte de pacientes críticos, sino como una opción que podría mitigar el daño por una desproporción en la oferta/demanda de la ventilación mecánica. Se presenta, a priori, como una estrategia puente hasta la llegada de los recursos apropiados, que concedería tiempo a pacientes que sin soporte ventilatorio no podrían sobrevivir. En el campo de la ética existen criterios que difieren en la selección de pacientes que deberían recibir el cuidado a expensas de limitárselo a otro paciente[1], particularmente en este caso el personal sanitario se enfrentaría al principio del mal menor. Entonces, las preguntas esenciales son, si frente a un escenario en donde un paciente necesita ventilación mecánica y no hay ventiladores disponibles, ¿conectarlo en paralelo a otro paciente bajo un único ventilador genera menos daño que no hacerlo?; ¿se debe limitar o compartir la ventilación mecánica? Esta revisión pretende analizar el "estado de arte" de esta posibilidad, presentar el modelo físico de su funcionamiento, describir su dinámica y llamar la atención sobre las advertencias.

\section{Antecedentes}

La idea de compartir la ventilación mecánica surge en contextos donde la capacidad del sistema sanitario de proveer un ventilador a cada paciente que lo necesite se ve comprometida. Ataques terroristas, desastres meteorológicos o epidemias son escenarios en donde esto sería posible[2].

\begin{tabular}{|c|c|c|c|}
\hline \multicolumn{2}{|c|}{ Mecánica } & \multicolumn{2}{|c|}{ Electricidad } \\
\hline Variable & Unidad & Variable & Unidad \\
\hline Volumen & $\mathrm{L}$ & Carga & Coulomb \\
\hline Flujo & $\mathrm{L} / \mathrm{s}$ & Corriente & Amperio \\
\hline Presión & $\mathrm{cmH} 2 \mathrm{O}$ & Voltaje & Voltio \\
\hline Resistencia & $\mathrm{cmH} 2 \mathrm{O} / \mathrm{L} / \mathrm{s}$ & Resistencia & Ohm \\
\hline Compliance & $\mathrm{L} / \mathrm{cmH} 2 \mathrm{O}$ & Capacitancia & Faradio \\
\hline
\end{tabular}

Figura 1. Esquema de conexión en paralelo.

Diferentes grupos[3]-[5] han explorado esta posibilidad con diferentes aproximaciones y conclusiones. En 2007, Neiman e Irvin[4] en un estudio piloto crean un modelo de conexión simple y de rápido ensamblaje con tubuladuras y tubos en T que permite ventilar simultáneamente dos pulmones artificiales (Figura 1). En la conclusión afirman que, ante un desastre, esa conexión podría representar una alternativa. Con una línea similar de trabajo y en el campo de la simulación, Branson et al.[5], generan un modelo parecido, pero modifican variables como resistencia y compliance de los pulmones artificiales, prueban diferentes modos ventilatorios y analizan los resultados cuantitativamente. El grupo de investigación expone las limitaciones de la estrategia de compartir la ventilación y se muestra escéptico sobre el papel que pudiese llegar a cumplir este método ante su necesidad real. Por otro lado, en 2008 surge un trabajo que procura determinar, con un modelo animal, si es posible ofrecer ventilación mecánica compartida a cuatro ovejas con un único ventilador[6]. Los animales fueron sedados, paralizados y ventilados durante doce horas. Se eligió esa cantidad de horas ya que es el tiempo que demorarían en llegar los recursos del "Strategic National Stockpile" en Estados Unidos, lugar donde se realizó el experimento. De una manera optimista concluyen que las ovejas pudieron ser mantenidas bajo ventilación mecánica durante ese tiempo sin un mayor compromiso de su estado. Su gran limitación es que utiliza pulmones sanos, y este podría no ser el escenario de una falla respiratoria masiva en la que se necesite la estrategia de compartir ventilación (excepto por botulismo, por ejemplo). Pacientes sépticos, quemados, con distrés pulmonar y/o descompensados hemodinámicamente, cuya problemática exceda la parálisis diafragmática, se comportarían de una manera diferente a las condiciones experimentales. En lo que respecta a lo más reciente escrito sobre la temática, entidades de gran valor científico como la American Society of Anesthesiologists (ASA) y la Society of Critical Care Medicine (SCCM), entre otras, emitieron una declara- 
ción conjunta sobre el tema[7]. En el artículo, sin hacer referencia a la cantidad de pacientes conectados, se desaconseja en general la práctica de compartir la ventilación mecánica, indicando que el ventilador debe otorgarse a quien tenga mayor probabilidad de beneficiarse. Se argumenta que conectar más de un paciente bajo un mismo ventilador genera mayor daño que limitar la ventilación de otro. Se citan los trabajos clásicos sobre el tema[4]-[6] y se argumentan los problemas típicos de esta práctica: contaminación cruzada, monitoreo dificultoso, implementación dificultosa, entre otros.

\section{Fundamento físico}

La función mecánica del sistema respiratorio puede ser modelada de diferentes formas[8], una de ellas es entenderlo como un circuito RC. Estos circuitos están compuestos por resistencias y capacitores conectados a una fuente de voltaje. En esta equivalencia, el flujo de gas equivale a la corriente, la presión al potencial, la resistencia al flujo a la resistencia eléctrica y la compliance a la capacitancia (Figura 2).

En el modelo más simple, la función mecánica pulmonar puede ser entendida como una resistencia y un capacitor conectados en serie, como muestra la Figura 3A. En dicho circuito, una diferencia de poten-

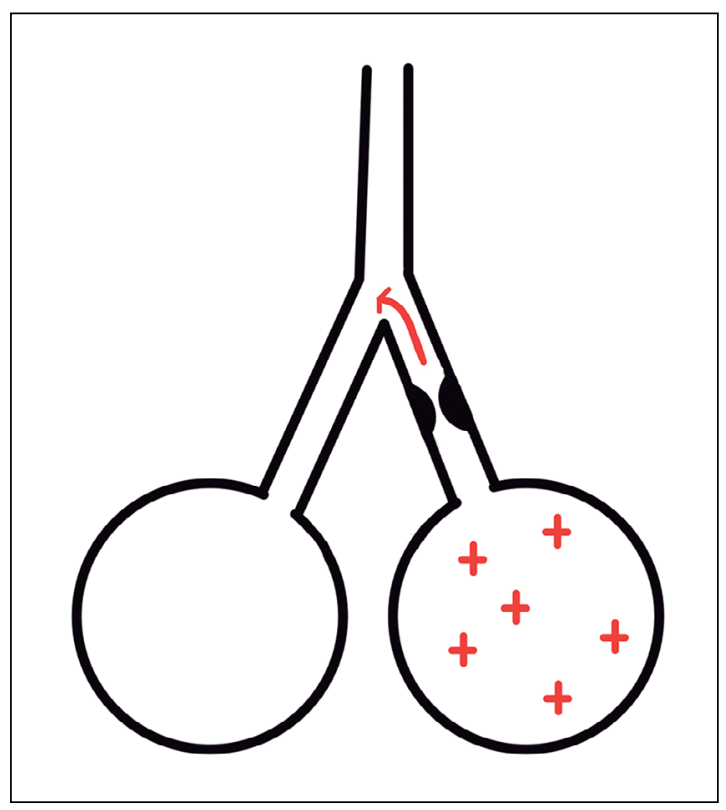

Figura 2. Analogía en sistemas mecánicos eléctricos. cial genera la circulación de corriente a través de la resistencia y carga el capacitor con una carga determinada. La corriente circulará hasta que el capacitor se cargue completamente para esa diferencia de potencial (la de la fuente), luego ya no se detectará corriente en ningún punto del circuito. La resistencia actúa como un disipador de potencial y disminuye el voltaje que recibe el capacitor para cargarse. El potencial disipado por la resistencia puede ser calculado a través de la ley de Ohm: (ecuación 1). De acuerdo a esta ley, el producto de la corriente que pasa por la resistencia y el valor de esta, determinarán la caída de potencial. El voltaje remanente será el que utilizará el capacitor para cargarse, que lo hará de acuerdo a la siguiente ecuación: (ecuación 2). Así, cuando mayor voltaje se le aplique y cuanto mayor sea su capacitancia (una propiedad inherente a cada capacitor), mayor será la carga obtenida.

Este modelo representa al pulmón como un capacitor y a la vía aérea de conducción como una resistencia, elementos que son sometidos a una diferencia de presión por el ventilador mecánico. De la misma manera que en el circuito eléctrico, en el circuito pulmonar existirá flujo hasta que el pulmón se cargue de

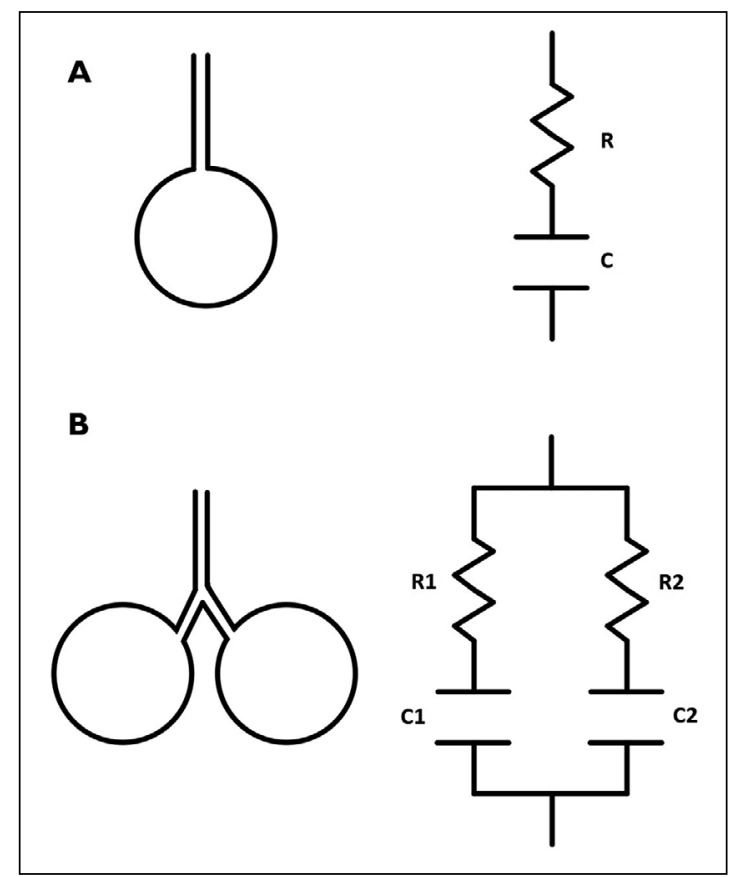

Figura 3. A. Analogía entre una unidad pulmonar y su correspondiente circuito eléctrico. B. Analogía entre dos unidades pulmonares conectadas en paralelo y su correspondiente circuito eléctrico. R: resistencia. C: capacitor. 
volumen para una diferencia de presión dada (la que genera el ventilador), luego ya no habrá flujo en el sistema. Podemos entender, de acuerdo a la ecuación 1 , que la vía de conducción (vía aérea instrumental y vía aérea anatómica) disipará presión de acuerdo al producto del flujo y la resistencia. De forma análoga, de acuerdo a la ecuación 2, el volumen insuflado en el pulmón dependerá de la presión a la que es sometido (que no es la configurada en el ventilador, sino la que quede luego de haber sido disipada una parte por la resistencia) y de su compliance (propiedad inherente al sistema toracopulmonar). De hecho, el volumen que ingresa a los pulmones puede ser entendido a través de la siguiente ecuación:

$$
\Delta P=(V / t) \times R+V \times(1 / C)
$$

Esta ecuación, denominada ecuación de movimiento del sistema respiratorio, muestra que, en su conjunto, las variables que determinan el volumen que ingresa a los pulmones son la resistencia, la compliance y la presión (siempre y cuando se le dé un tiempo suficiente, ver más adelante constante de tiempo).

Un análisis similar se puede hacer formando un circuito con resistencias y capacitores en paralelo, según la Figura 3B. En este circuito, cada componente RC está sometido a la misma diferencia de voltaje, y la carga de cada capacitor dependerá del voltaje al que es sometido (como se ha dicho, igual en ambos), su propia capacitancia y la resistencia que tiene por detrás. La carga total del sistema es la suma de las cargas individuales; de la misma manera, la capacitancia total del sistema es la suma de las capacitancias individuales. Este simple circuito puede representar el modelo con el que se interpreta la distribución de la ventilación entre dos alvéolos pulmonares, dos lóbulos pulmonares, dos pulmones del mismo paciente o, por qué no, entre dos pacientes conectados al mismo ventilador.

Gracias al modelo, podemos entender qué sucede cuando la ventilación mecánica se comparte. En primer lugar, como la capacitancia del circuito es la suma de ambas, la capacitancia total es mayor a las individuales, por lo que debemos contar con una fuente capaz de asegurar un voltaje de carga constante frente a una demanda de carga mayor. Esto nos muestra que el sistema de dos pacientes conectados a un ventilador tendrá mayor compliance que cada paciente particular, y que el ventilador mecánico debería ser capaz de presurizar un sistema más complaciente y con una demanda de volumen mayor.

En segundo lugar, la carga de cada capacitor será independiente de la carga del otro. Así, en ventilación a presión control, el volumen corriente de un paciente es independiente de las condiciones del otro.

En tercer lugar, dado un voltaje, las características intrínsecas de cada grupo RC que determinan su carga son su capacitancia y su resistencia, y la carga total del sistema es la suma de las cargas individuales. Esto se traduce, en ventilación mecánica en presión control, a que el volumen insuflado en cada paciente (dada una driving pressure y un tiempo inspiratorio) depende solo de su propia compliance y resistencia, y que el volumen total insuflado por el ventilador es la suma del volumen insuflado a cada paciente.

Otro punto a tener en cuenta es la constante de tiempo de cada componente RC. Esta variable da cuenta del retraso que hay entre un input y un output en un sistema; en este caso, el tiempo entre que se aplica el voltaje y el capacitor se carga. La constante de tiempo $(\tau)$ de cada componente RC es el producto de su resistencia y su capacitancia, se expresa en unidades de tiempo y refleja la velocidad de carga (o descarga). Al pasar el tiempo de una constante de tiempo, el capacitor se cargará un $63,2 \%$; al pasar dos un $86,5 \%$; tres un $95 \%$ y así sucesivamente mostrando un comportamiento asintótico hacia la carga máxima para ese voltaje. En fisiología pulmonar, el mismo concepto puede aplicarse y la constante de tiempo se calcula, de manera análoga, como el producto de resistencia y compliance. Esto nos muestra que, dado un sistema respiratorio, si en el lapso en el que este es expuesto a una diferencia de presión, no se dejan pasar al menos cinco constantes de tiempo, el volumen insuflado no será el máximo (para esa diferencia de presión). En términos prácticos, si no se alcanzan al menos cinco constantes de tiempo durante el tiempo inspiratorio, el flujo inspiratorio no llegará a cero al final de la inspiración. Como contraparte, si durante el tiempo espiratorio no se alcanzan las cinco constantes de tiempo, a la llegada de la siguiente inspiración el pulmón todavía conservará volumen del ciclo anterior, apareciendo el atrapamiento aéreo o auto-PEEP. Eventualmente, el sistema tenderá al equilibrio, ya que el volumen atrapado disminuirá paulatinamente la compliance pulmonar, lo cual disminuirá la constante de tiempo del sistema y entonces el pulmón podrá vaciarse en el tiempo espiratorio a expensas de un volumen mayor.

En ventilación compartida, este fenómeno cobra relevancia dado que la constante de tiempo de cada paciente es diferente, lo que implica que puede darse el caso en el que el tiempo espiratorio (igual para ambos) no es suficiente para que uno de los pacientes complete su vaciado pulmonar y el otro sí. En esta situación, al final de la espiración podría existir flujo 
entre los pacientes, desde el pulmón con constante de tiempo mayor al de menor[9], como representa la Figura 4. Este hecho representaría una variable del pendelluft, pero que en este caso sucedería entre dos pacientes[10]. Este fenómeno se vería exagerado al disminuirse el tiempo espiratorio (por ejemplo, al aumentar la frecuencia respiratoria con relación I:E constante) y podría ser relevante en términos de contaminación cruzada.

El modelo eléctrico para entender la mecánica pulmonar tiene sus limitaciones, como por ejemplo que el pulmón no se comporta estrictamente como un capacitor ideal ya que no es capaz de devolver el $100 \%$ de su carga en forma de corriente, sino que disipa energía en el proceso (evidenciado por el fenómeno de histéresis)[11]; o que la complejidad de la ventilación mecánica, por su impacto hemodinámico y condiciones dinámicas excede al modelo. Sin embargo, este análisis puede servir para entender que lo mismo que sucede al conectar dos pacientes a un ventilador puede estar sucediendo en la distribución de la ventilación entre ambos pulmones de un único paciente, entre lóbulos de un pulmón, o incluso, de manera fractal y a menor escala, en la ramificación de la vía aérea.

\section{Dinámica de la ventilación compartida}

Conectar más de un paciente a un ventilador genera un cambio de concepto en la ventilación mecánica, donde ahora son los pacientes los que deben ser adaptados al ventilador y no al revés. Los pacientes están expuestos a una única configuración ventilatoria y comparten valores como la $\mathrm{FiO}_{2}$ y la PEEP, pero no otros, como el volumen corriente o flujo. El monitoreo individual ya no puede realizarse por la interpretación de las curvas del ventilador (que ahora describen al grupo), sino por otros medios como la saturación periférica $\left(\mathrm{SpO}_{2}\right)$, capnografía y medición de gases en sangre. Por su parte, la configuración de alarmas sumaría otro reto: el ventilador solo podría alertar ante un problema con el grupo y no de un paciente en particular.

La conexión de los pacientes al ventilador es en paralelo, es decir, están sometidos a la misma presión en la tubuladura inspiratoria y el volumen que ingrese a cada uno dependerá de su propia compliance y resistencia, esto es independiente al modo ventilatorio. Se debería evitar cualquier tipo de perturbación de los pacientes al ventilador, ya que la desadaptación de uno comprometería al otro. Si, por ejemplo, un

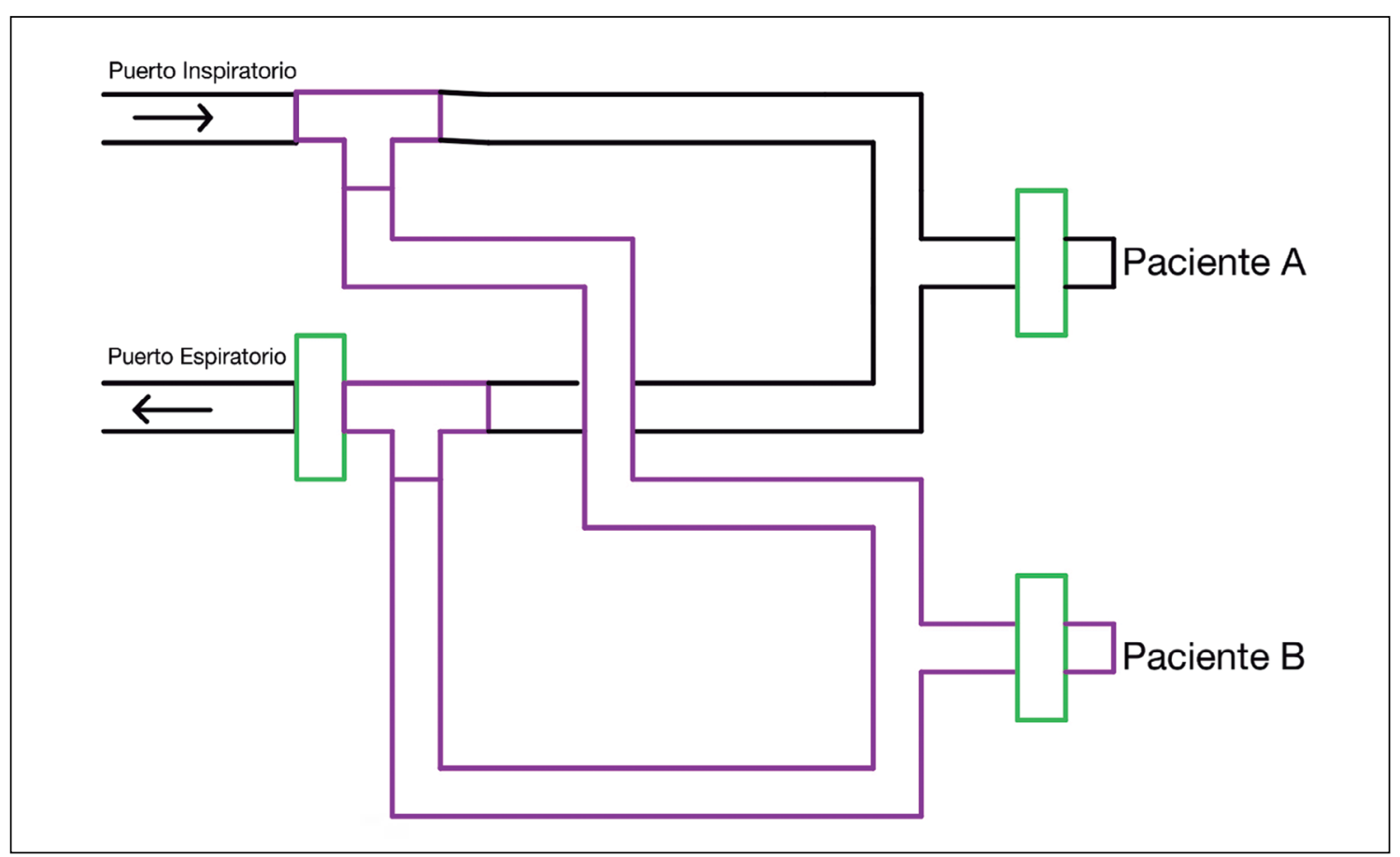

Figura 4. Dos unidades pulmonares con diferentes constantes de tiempo $(\tau)$ conectadas en paralelo a fin de espiración. La flecha indica la dirección del flujo que podría existir, desde la unidad con mayor $\tau$ a la de menor. 
paciente gatilla una respiración mandatoria, ambos serían ventilados. Estas perturbaciones se evitan por un lado con la configuración del ventilador (quitando la posibilidad de gatillo o configurando un umbral muy alto) y, por otro lado, con niveles de sedación/ parálisis adecuados.

Si bien es esperable que la alta demanda por la ventilación mecánica siga a una única injuria (trauma, enfermedad infectocontagiosa, etc.) y, por lo tanto, el patrón de daño pulmonar sea similar (lo que facilitaría el agrupamiento por estadio de daño), cada paciente empeora o mejora a diferente ritmo, lo que en la práctica también sumaría dificultad al momento de parearlos.

En cuidados intensivos es vital adelantarse a eventos críticos, y el paro cardiorrespiratorio presentaría desafíos particulares en caso de que se presente en pacientes que comparten la ventilación mecánica: ¿cómo se debería continuar la ventilación?; ¿cómo se previene la aerosolización si se ventila con bolsa autoexpandible? Eventos así resaltan la importancia de la protocolización en medicina crítica.

Con lo que respecta al modo ventilatorio, y desde un punto de vista teórico, la ventilación controlada por presión (PCV) presenta varias ventajas frente a la controlada por volumen (VCV) por varias razones: 1) cambios en la compliance y/o resistencia de un paciente no alterarán al otro; 2 ) se evita la necesidad de que los pacientes sean de tamaño similar, ya que no es necesario ajustar un volumen corriente al peso del grupo. Los pulmones más grandes tienen mayor compliance absoluta, por lo que recibirán mayor volumen corriente; 3) En PCV se configura la presión de trabajo, pero no se puede garantizar el volumen entregado, fenómeno característico de la ventilación por presión e independiente de la cantidad de pacientes conectados; en VCV no se controla ni la presión en el sistema ni el volumen corriente a cada pulmón (sí al conjunto de ambos pacientes); 4) Como el sistema de tubuladuras es de mayor volumen, su compliance absoluta es mayor, si esto no es compensado, disminuye el volumen corriente que llega a los pacientes en VCV, no así en PCV; 5) En PCV, el tamaño del tubo endotraqueal de cada paciente es indiferente para el otro, sólo variará la resistencia del propio individuo ventilado; si la ventilación es controlada por volumen, la resistencia del tubo sí definirá la distribución del volumen configurado al grupo. También se debe tener en cuenta que, si el ventilador se programa en VCV, el volumen corriente que se configura se entrega al sistema, y cada paciente recibirá una parte de acuerdo a su compliance y resistencia, por lo tanto, si un tubo se obstruye o la compliance de un paciente disminuye, su volumen recibido disminuirá y el pulmón del otro paciente se verá sometido a volúmenes corrientes altos. En VCV los cambios de un paciente sí afectan al otro. En PCV los cambios de un paciente sólo afectan ventilatoriamente al paciente que los sufre. Sí existen características que podrían generar diferencias en la distribución de la ventilación que harían no apto a ese conjunto de pacientes: obesidad, disminución de la compliance pulmonar por progreso de la enfermedad, embarazadas, broncoespasmo, etc.

Una maniobra que permite aislar a un paciente del otro y cuantificar cada variable ventilatoria de manera personalizada es clampear el tubo endotraqueal de uno de los pacientes por pocos ciclos ventilatorios. Es importante estar ventilando en modo controlado por presión, así el hecho de "privar" de ventilación al paciente que se separa no influye en el otro. Durante la maniobra puede ponerse en evidencia el volumen corriente, la compliance, la morfología de las curvas y varios datos más que sólo corresponden al paciente ventilado, y que al volver a la ventilación doble seguirán siendo los mismos (sólo que ya no mostrados).

En términos de implementación, y desde un punto de vista puramente físico, conectar 2, 4 o 6 pacientes a un ventilador no implica demasiada diferencia. En teoría, una conexión en paralelo lograría que cada paciente sea independiente de los otros y solo se necesitaría un ventilador capaz de presurizar un sistema de mayor tamaño. En un contexto real, esto es en extremo difícil de aplicar, ya que existirán limitaciones operativas del ventilador, diferencias de compliance, resistencia, estado hemodinámico de los pacientes y necesidad de monitoreo personalizado que volverían caótica la implementación.

Teniendo en cuenta lo anterior, es claro que la calidad de la ventilación es mucho menor a la que existiría si la configuración ventilatoria fuera única y personalizada. Si muchas veces ventilar un pulmón enfermo y no homogéneo presenta dificultades, compartir la ventilación en dos pacientes puede generar problemas mayores. Esto no implica que al menos no deba plantearse la cuestión ética del mal menor: recordar que el objetivo de unir dos pacientes bajo un mismo ventilador es ganar tiempo hasta la llegada de recursos y dañar lo menos posible.

\section{Contaminación cruzada}

Un punto importante es el de la contaminación cruzada de pacientes y del medio en el que los pacientes son ventilados. Con respecto a la primera, 
si bien la dirección del flujo es unidireccional, y los pacientes conectados compartirán el patógeno causante de la enfermedad, se deberían balancear riesgos y beneficios de manera particular. Por otro lado, ante un inadecuado nivel de sedación, si un paciente aplica presión negativa al circuito inspiratorio, robaría gas del otro paciente, presentándose contaminación cruzada[5].

Con respecto a la ventilación manual, si bien surge como una alternativa a la alta demanda de ventilación mecánica[12], esto acarrea sus propias complicaciones como la necesidad de mayor recurso humano y la exposición a contagios tanto de los pacientes como de los operadores[13].

En relación a la salida de gases del ventilador, debería considerarse el caso de que pequeñas gotas contengan microorganismos capaces de transmitir la enfermedad. Esto fue investigado durante el brote de SARS en 2003 y si bien no se demostró como un mecanismo de transmisión, deberían tomarse precauciones ${ }^{14}$. La forma de evitar este fenómeno es por medio de la colocación de filtros antibacteriales al final de la tubuladura espiratoria y entre el tubo endotraqueal y la pieza en $Y$, protegiendo la toma de muestra del capnógrafo.

\section{Conclusiones}

Compartir la ventilación mecánica surge como una alternativa ante la posibilidad de que un paciente no tenga acceso a un ventilador individual. Esta opción tiene implicancias técnicas, sanitarias, éticas y legales. Aunque la evidencia es limitada, existen argumentos y recomendaciones en contra de su implementación. Por el momento, no contamos con la rigurosidad científica para afirmar que el daño de no ventilar a un paciente que lo necesite es menor que acoplarlo a otro paciente. Esperamos que este aporte sea de relevancia y sirva como referencia a futuros trabajos de mayor calidad científica.

\section{Financiamiento}

Los autores declaran que no hubo financiamiento.

\section{Conflicto de intereses}

Los autores declaran que no hay conflicto de intereses.

\section{Responsabilidades éticas}

Protección de personas y animales. Los autores declaran que para esta investigación no se han realizado experimentos en seres humanos ni en animales.

Confidencialidad de los datos. Los autores declaran que para esta investigación no se han recabado datos de pacientes.

Derecho a la privacidad y consentimiento informado. Los autores declaran que en este artículo no aparecen datos de pacientes.

\section{Referencias}

1. White $\mathrm{DB}$, Katz $\mathrm{MH}$, Luce JM, Lo $B$. Who should receive life support during a public health emergency? Using ethical principles to improve allocation decisions. Ann Intern Med. 2009 Jan;150(2):132-8. https:// doi.org/10.7326/0003-4819150-2-200901200-00011 PMID:19153413

2. Rubinson L, O'Toole T. Critical care during epidemics. Crit Care. 2005 Aug;9(4):311-3. https://doi.org/10.1186/cc3533 PMID:16137366

3. Darowski M, Englisz M. Artificial ventilation of the lungs for emergencies. Front Med Biol Eng. 2000;10(3):177-83. https:// doi.org/10.1163/156855700520 62576 PMID:11014679

4. Neyman G, Irvin CB. A single ventilator for multiple simulated patients to meet disaster surge. Acad Emerg Med. 2006 Nov;13(11):1246-9. https://doi. org/10.1197/j.aem.2006.05.009 PMID:16885402

5. Branson RD, Blakeman TC, Robinson BR, Johannigman JA. Use of a single ventilator to support 4 patients: laboratory evaluation of a limited concept. Respir Care. 2012
Mar;57(3):399-403. https://doi. org/10.4187/respcare.01236 PMID:22005780

6. Paladino $L$, Silverberg $M$, Charchaflieh JG, Eason JK, Wright BJ, Palamidessi N, Arquilla B, Sinert R, Manoach $S$. Increasing ventilator surge capacity in disasters: ventilation of four adulthuman-sized sheep on a single ventilator with a modified circuit. Resuscitation 2008; 77(1):121; PMID:18164798; . resuscitation.2007.10.016. https://doi.org/10.1016/j. resuscitation.2007.10.016.

7. "Joint Statement on Multiple 
Patients Per Ventilator." Anesthesia Patient Safety Foundation, 27 Mar. 2020, www.apsf.org/news-updates/ joint-statement-on-multiplepatients-per-ventilator/

8. Ghafarian P, Jamaati $\mathrm{H}$, Hashemian SM. A Review on Human Respiratory Modeling. Tanaffos. 2016;15(2):61-9. PMID:27904536

9. Otis $A B$, McKerrow $C B$, Bartlett RA, Mead J, Mcllroy $M B$, Selver-Stone NJ, et al. Mechanical factors in distribution of pulmonary ventilation. J Appl Physiol. 1956 Jan;8(4):427-43. https://doi. org/10.1152/jappl.1956.8.4.427

\section{PMID:13286206}

10. Greenblatt EE, Butler JP, Venegas JG, Winkler T. Pendelluft in the bronchial tree. J Appl Physiol (1985). 2014 Nov;117(9):979-88. https://doi.org/10.1152/ japplphysiol.00466.2014 PMID:25170072

11. Escolar JD, Escolar A. Lung hysteresis: a morphological view. Histol Histopathol. 2004 Jan;19(1):159-66. PMID:14702184

12. West JB. The physiological challenges of the 1952 Copenhagen poliomyelitis epidemic and a renaissance in clinical respiratory physiology.
J Appl Physiol (1985).

2005 Aug;99(2):424-32.

https://doi.org/10.1152/

japplphysiol.00184.2005

PMID:16020437

13. Weber DJ, Wilson MB, Rutala WA, Thomann CA. Manual ventilation bags as a source for bacterial colonization of intubated patients. Am Rev Respir Dis. 1990 Oct;142(4):892-4. https://doi. org/10.1164/ajrccm/142.4.892 PMID:2221598

14. ECRI Institute. Mechanical ventilation of SARS patients: lessons from the 2003 SARS outbreak. Health Devices. 2020 Feb. 